\title{
Subcellular localization of the ABCG2 transporter in normal and malignant human gallbladder epithelium
}

\author{
Sylvia Aust ${ }^{1}$, Peter Obrist ${ }^{2}$, Walter Jaeger ${ }^{3}$, Martin Klimpfinger ${ }^{4}$, Gerhard Tucek $^{4}$, \\ Friedrich Wrba ${ }^{5}$, Edward Penner ${ }^{6}$ and Theresia Thalhammer ${ }^{1}$ \\ ${ }^{1}$ Department of Pathophysiology, Medical University of Vienna; ${ }^{2}$ Department of Pathological Anatomy, \\ University of Innsbruck; ${ }^{3}$ Institute of Pharmaceutical Chemistry, University of Vienna; ${ }^{4}$ Department of \\ Pathology, Ludwig Boltzmann Institute of Applied Molecular Pathology, Kaiser-Franz-Josef-Spital; \\ ${ }^{5}$ Department of Clinical Pathology and ${ }^{6}$ Clinic of Internal Medicine, Medical University of Vienna, \\ Vienna, Austria
}

\begin{abstract}
Epithelium of the gallbladder and biliary tract is exposed to high concentrations of potentially harmful exogenous and endogenous compounds excreted into primary bile. As the ATP-dependent efflux pump ABCG2 can prevent cellular accumulation of anticancer drugs, estrogen sulfate, xenobiotics, porphyrins, and sterols, its expression in the biliary tract might mediate protection by hindering their penetration. We therefore investigated the expression and subcellular distribution of ABCG2 in normal and malignant human gallbladder. After demonstrating ABCG2 expression in gallbladder epithelium by RT-PCR and Western blotting, we analyzed the subcellular localization of ABCG2 by indirect immunofluorescence in gallbladder adenocarcinoma specimens, and compared it to that in cholelithiasis, and normal gallbladder samples $(n=54)$. In control, cholelithiasis, and well-differentiated tumor samples (grade 1, T1-3), ABCG2 is present at the luminal membrane of epithelial cells, which was proven by colocalization of apical-bound TRITC-labeled lectin (wheat germ agglutinin). In poorly differentiated gallbladder adenocarcinomas, intracellular ABCG2, in addition to luminal ABCG2 immunoreactivity, was found in 13/21 carcinoma samples (grade 2 and 3, T2-4, $P<0.01$ ). In 3/11 of grade 3 tumors, ABCG2 was present in the cytoplasmatic compartment only $(P<0.01)$. In proliferating bile ducts of cholangiocarcinomas, ABCG2 showed an analogous staining pattern with presence in cytosolic compartments. However, the apical marker enzyme neutral endopeptidase remained on the membrane in all samples. To study whether phosphatidylinositol 3-kinase (PI3K) signaling might be necessary for ABCG2 membrane insertion, we treated freshly isolated human gallbladder epithelial cells with the PI3K inhibitor wortmannin. As assessed by indirect immunofluorescence, this maneuver redistributes ABCG2 to intracellular compartments. In conclusion, our data suggest a protective role for ABCG2 in well-differentiated gallbladder epithelial cells. Cytoplasmatic accumulation of ABCG2 in poorly differentiated carcinomas might coincide with malfunctioning of PI3K-signaling pathways during tumor progression.
\end{abstract}

Laboratory Investigation (2004) 84, 1024-1036, advance online publication, 7 May 2004; doi:10.1038/labinvest.3700127

Keywords: ABCG2; gallbladder epithelium; gallbladder carcinoma; cholelithiasis; phosphatidylinositol 3-kinase; BCRP; neutral endopeptidase

Cholelithiasis, and as a complication, acute cholecystitis, are among the most common gastrointestinal diseases in industrialized countries, while carcinomas of the gallbladder are quite rare. Although cholelithiasis is considered an important

Correspondence: Dr T Thalhammer, PhD, Department of Pathophysiology, Medical University of Vienna, AKH, Waehringer Guertel 18-20, A-1090 Vienna, Austria.

E-mail: Theresia.Thalhammer@akh-wien.ac.at

Received 19 December 2003; revised 8 April 2004; accepted 20 April 2004; published online 17 May 2004 risk factor for gallbladder carcinoma, only $1-2 \%$ of patients who have operations for gallstones are diagnosed with gallbladder cancer at the time of surgery. The epidemiological characteristics of cholelithiasis and gallbladder cancer are closely related. Risk factors associated with the formation of cholesterol gallstones including estrogen exposure, nutritional factors, obesity, as well as age, ethnic background, abnormalities in the biliary tract, etc, are also associated with an increased risk of gallbladder carcinoma development. ${ }^{1}$ Additionally, chemical carcinogens, either of nutritional or 
occupational origin, may contribute to the development of this malignancy in humans, and in fact, an increased risk of gallbladder cancer was found in people employed in petroleum refining, paper mills, chemical processing, and textile work. ${ }^{2}$ However, the etiology of gallbladder carcinoma is not yet fully elucidated, and, moreover, despite improvements in diagnosis, surgery, and chemotherapy, the prognosis of gallbladder cancer is still poor. Therefore, it is of great importance to identify mechanisms that could be involved in protecting tissues in the biliary tract against harmful compounds of endogenous or exogenous origin in bile.

In various tissues and organs, members of the ATP-binding cassette (ABC) transporter family serve as export pumps for cytotoxic drugs, environmental agents, and toxins as wells as endogenous compounds including hormones. Since they limit the intracellular accumulation of these substrates, they prevent harmful effects on cells and organs. ${ }^{3}$ In liver, the ABC export pumps P-glycoprotein (Pgp, MDR1 gene product, ABCB1) and the multidrug resistancerelated protein 2 (MRP2, ABCC2, cMOAT) effectively extrude xenobiotics and breakdown products from endogenous compounds through the canalicular membrane of the hepatocyte into the primary hepatic bile. ${ }^{4,5}$ On its way from the canalicular lumen down to the intestine, primary bile is modified by epithelial cells lining the bile ducts. ${ }^{6}$ Therefore, these epithelial cells are exposed to high concentrations of various, potentially toxic compounds present in bile. As a result, the concentration gradient between the lumen and intracellular space supports their reuptake into epithelial cells. For that reason, powerful efflux pumps are required to prevent penetration of these compounds into the tissue. These mechanisms are of particular importance for the gallbladder epithelium, where bile is concentrated and stored for a considerable period of time. In fact, Pgp and MRP2 have been identified in the apical membrane of gallbladder epithelium, ${ }^{7,8}$ and cholangiocytes, ${ }^{9}$ where they may limit the penetration of bile-borne compounds.

Recently, the ATP-dependent efflux pump ABCG2, originally cloned from a breast cancer cell line and called 'breast cancer resistance protein', ${ }^{10}$ gained much interest as a protective factor positioned at various strategic sites inside the body. Located at the apical membrane of epithelial cells in the large and small intestine, the renal proximal tubulus, hepatocyte canalicular membranes, the blood-brain barrier, and placental syncytiotrophoblasts, it limits the uptake of drugs, hormones, and xenobiotics into the circulation, bile, brain, and fetus, respectively. ${ }^{11}$ It is expressed in stem cells in blood and organs (liver oval cells) providing protection against toxins, thereby, supporting their survival. ${ }^{12,13}$

ABCG2 is a half-transporter, exhibiting a broad substrate specificity for various anticancer agents, including mitoxantrone, anthracyclines, and camp- tothecins. ${ }^{14,15,16}$ It is implicated in the transport of endogenous compounds, for example, sulfate conjugates of estrogen, ${ }^{17}$ and its expression can be modified by estrogen. ${ }^{18}$ Expression of the transporter also protects against a certain form of porphyria characterized by severe phototoxicity, which was observed in ABCG2 knockout mice after exposure to a diet rich in the chlorophyll-derived phototoxin pheophorbide and UV light. ${ }^{19,20}$ In these ABCG2 knockout mice, the transporter also limits the exposure to dietary-derived heterocyclic amines, which act as carcinogens and which are widely present in protein-rich foods and cigarette smoke. ${ }^{21}$ Also, unconjugated bilirubin has been proposed as a substrate for ABCG2. ${ }^{22}$ Recently, it has been discovered that ABCG2 also mediates sterol transport. ${ }^{23}$ Therefore, ABCG2 could work in concert with other half-transporters of the ABCG family implicated in sterol transport, namely ABCG5 and 8, which have recently been identified in gallbladder epithelium. ${ }^{24}$ Moreover, ABCG2 can alter the organization of phospholipids in the plasma membrane, which in turn may influence cell surface properties and cellcell interactions. ${ }^{25}$

The function of ABCG2 as an export pump for anticancer drugs and xenotoxins is consistent with a subcellular localization in the plasma membrane as demonstrated in normal and tumor cells as well as in various tumor cell lines. ${ }^{10,11,26}$ Although ABCG2 confers multidrug resistance to anticancer drugs in various tumor cell lines, ${ }^{27}$ its implication in drug resistance in tumor patients is not yet fully elucidated. While in most tumors, ABCG2 is located in the membrane, additional cytoplasmic ABCG2 staining is occasionally seen, for example, in hepatocellular carcinoma samples, ${ }^{28}$ and even in some ABCG2 overexpressing tumor cell lines, cytoplasmatic staining is seen as well. ${ }^{29}$ On the other hand, in malignant tissues like the urinary bladder, ABCG2 is mainly found in cytoplasmatic compartments. ${ }^{30}$

Although ABCG2 is highly expressed in some tumors, for example, lung cancer, in other tumors, for example, breast cancer, its expression is quite low compared to normal ductal breast tissue. ${ }^{31}$ Therefore, it is not clear whether ABCG2 confers resistance to anticancer drugs in breast cancer patients. It is more likely that ABCG2 has a protective function in normal tissue and this may be lost in this malignancy. Vanishing of ABCG2 seems to mark the loss of differentiation. ${ }^{32}$

The reason for the alterations in the subcellular ABCG2 location in tumors is unknown. Signaling by the phosphatidylinositol 3-kinase (PI3K)/Akt pathway might regulate insertion of ABCG2 into the plasma membrane. This is proposed from experiments in human side population cells of the hematopoietic system, in which application of a PI3K inhibitor results in a redistribution of ABCG2 from the membranes into the intracellular compartment. ${ }^{33}$ Consistent with the role of PI3K in the 
regulation of the subcellular localization of ABCtransporters, in rat liver cells, the transport of newly synthesized ABC-transporters, for example, Pgp and mrp2, to the canalicular membrane, and the functioning of these export pumps at the canalicular liver cell membrane, depend on PI3K signaling. ${ }^{34}$ This pathway could also play a role in the insertion of ABC-transporters into the membrane of other epithelial cells including gallbladder.

Regarding the significant impact that ATP-dependent efflux pumps such as ABCG2 might have on the protection of the biliary epithelium, we investigated the expression of this transporter in the healthy and diseased gallbladder. Furthermore, we determined whether membrane insertion of the transporter is dependent on PI3K signaling.

\section{Materials and methods}

\section{Tissues and Tumor Samples}

All samples were obtained with the patients' written formal consent according to the guidelines of the ethical committee of the institution.

Normal gallbladder samples $(n=3 ; 1$ female/2 males) were derived from healthy liver donors (liver transplantation). The gallbladder was removed before livers were transplanted into the recipients. Cholelithiasis samples were obtained from 10 patients (seven females/three males), who underwent cholecystectomy for symptomatic gallstone disease. Chronic inflammatory lesions were seen in all cases. In all, 41 patients (30 females/11 males) with an adenocarcinoma of the gallbladder (TNM classification according to the International Union against Cancer (UICC) and the American Joint Committee on Cancer (AJCC) ${ }^{35}$ ) underwent either radical cholecystectomy, where no macro- or microscopic residual tumor was left behind (R0), or a palliative resection of the tumor (R1). Overall, 20 carcinomas were classified as well-differentiated (G1), 10 as moderately differentiated (G2), and 11 as poorly differentiated (G3). Clinical characteristics of patients are summarized in Table 1.

Additional tumor samples were obtained from two patients with moderately differentiated and two with poorly differentiated cholangiocarcinoma (CCC). Normal specimens from extrahepatic bile ducts and livers $(n=3$, each) were derived from autopsy specimens within $12 \mathrm{~h}$ after death (patients without liver and gallbladder disease).

For RT-PCR, Western blot and immunofluorescence studies (cryosections), tissue samples were snap-frozen and stored at $-80^{\circ} \mathrm{C}$ until use. For isolation of gallbladder epithelial cells, surgical samples were kept in Leibovitz L-15 cell culture medium before cell isolation was carried out (within $2 \mathrm{~h}$ after removal).

Paraffin sections (fixed with $4.5 \%$ formaldehyde) from patients' samples were subjected to routine pathological examination. Parts of these specimens, which were not required for further diagnosis, were used for investigations.

\section{RT-PCR}

Total RNA was extracted either from frozen tissue or freshly isolated GBEC with TRIzol reagent (Invitrogen, Paisley, Scotland). cDNAs were prepared according to standard methods. The following intron-spanning primers were used: ABCG2 (231 bp): forward: 5'-CAA CCA TTG CAT CTT GGC TG-3', reverse: $5^{\prime}$-CAA GGC CAC GTG ATT CTT CC$3^{\prime} ;{ }^{11}$ CK19 (433 bp): forward: 5'-GTG GAG GTG GAT TCC GCT CC-3' ${ }^{\prime}$, reverse: $5^{\prime}$-TGG CAA TCT CCT GCT CCA GC- $3^{\prime}$. $\beta$-actin served as an endogenous control. PCR conditions: denaturation at $94^{\circ} \mathrm{C}$ for $30 \mathrm{~s}$, annealing at $54^{\circ} \mathrm{C}$ (ABCG2) or $58^{\circ} \mathrm{C}$ (CK 19) for

Table 1 Characteristics of patients with gallbladder cancer $(n=41 ; \mathrm{F} / \mathrm{M} 31 / 10)$

\begin{tabular}{|c|c|c|c|c|c|}
\hline T classification & $1 a$ & $1 b$ & 2 & 3 & 4 \\
\hline \multicolumn{6}{|l|}{ Grade 1} \\
\hline $\mathrm{F} / \mathrm{M}$ & $6 / 0$ & $9 / 2$ & $1 / 1$ & $0 / 1$ & - \\
\hline Age & 74.3 & 65 & 66.5 & 79 & - \\
\hline Survival & $54.7(41-89)$ & $52(1-144)$ & $9.5(6-13)$ & 14 & - \\
\hline R0/R1 & $5 / 1$ & $6 / 5$ & $1 / 1$ & $0 / 1$ & - \\
\hline \multicolumn{6}{|l|}{ Grade 2} \\
\hline F/M & - & - & $5 / 3$ & $0 / 1$ & $1 / 0$ \\
\hline Age & - & - & 70.5 & 62 & 69 \\
\hline Survival & - & - & $9.5(3-136)$ & 23 & 3 \\
\hline R0/R1 & - & - & $2 / 6$ & $0 / 1$ & $1 / 0$ \\
\hline \multicolumn{6}{|l|}{ Grade 3} \\
\hline F/M & - & $1 / 0$ & $1 / 2$ & $2 / 1$ & $4 / 0$ \\
\hline Age & - & 78 & 73 & 69 & 71 \\
\hline Survival & - & 79 & $13.7(6-20)$ & $3.3(1-5)$ & $5(1-10)$ \\
\hline R0/R1 & - & $1 / 0$ & $1 / 2$ & $0 / 3$ & $0 / 4$ \\
\hline
\end{tabular}

F, female; M, male.

For age (years) and survival (months) of patients, median values are indicated. Additionally, minimum and maximum survival is also indicated.

Residual tumor stage: R0/R1, complete/incomplete tumor resection. 
$1 \mathrm{~min}$ and extension at $72^{\circ} \mathrm{C}$ for $1 \mathrm{~min}, 35$ cycles. For negative controls, the PCR reaction was performed without prior reverse transcription. Amplified cDNA was visualized by ethidium bromide staining on $2 \%$ agarose gels on a Herolab gelscanner (Wiesbach, Germany).

\section{Antibodies and Fluorescent Probe}

The monoclonal antibody against ABCG2, BXP-21 (IgG2a), recognizes a 126 amino-acid peptide subsequence (aa 271-396) of the transporter and was purchased from Signet (Dedham, MA, USA). The monoclonal antibody against neutral endopeptidase (anti-CD 10/CALLA Ab-2) and the monoclonal antibody against cytokeratin 19 (clone A53-B/A2.26) were distributed by Lab Vision-Neo Markers (Fremont, CA, USA). Fluorescence dye-labeled secondary antibodies were obtained from Molecular Probes (Eugene, OR, USA). TRITC-labeled wheat germ agglutinin (TRITC-WGA) was obtained from Sigma (Munich, Germany).

\section{Western Blot}

Tissues and isolated GBEC were homogenized in ice-cold lysis buffer (50 mM Tris-HCl, $\mathrm{pH}$ 7.4, $1 \mathrm{mM}$ EDTA, $2 \mathrm{mM}$ EGTA, $1 \mathrm{mM}$ dithiothreitol, $1 \mathrm{mM}$ phenylmethylsulfonyl fluoride, 1\% Nonidet NP-40, and combined phosphatase/protease inhibitor cocktail (Sigma, St Louis, MO, USA)). After centrifugation of the crude homogenate at $1500 \times g$ for 5 min to remove the cell nuclei, the supernatant was stored at $-80^{\circ} \mathrm{C}$ until use. Protein contents were determined by a kit from Biorad based on the Lowry method (Hercules, CA, USA).

Samples containing $100 \mu \mathrm{g}$ protein were subjected to electrophoresis on $10 \%$ polyacrylamide gels. Proteins were transferred electrophoretically onto polyvinylidene fluoride (PVDF) membranes (Pall Life Sciences, Ann Arbor, MI, USA). After blocking, blots were probed with antibodies against ABCG2 and CK19 (0.25 and $0.2 \mu \mathrm{g} / \mathrm{ml}$, respectively). Horseradish peroxidase-conjugated isoform-specific IgGs (Jackson Immuno Research Laboratories Inc., West Grove, PA, USA) were used as secondary antibodies. Immunoreactive proteins were visualized on Kodak X-ray films after treatment of membranes with the SuperSignal ${ }^{\circledR}$ chemiluminescent substrate (Pierce, Rockford, IL, USA).

\section{Indirect Immunofluorescence and TRITC-WGA Labeling}

Paraffin-embedded tissue sections, $4.5 \%$ formaldehyde-fixed, were deparaffinized in xylene, and rehydrated with decreasing concentrations of ethanol according to standard methods. After a permeabilization step (0.1\% PBS-Tween 20, Sigma), which was introduced to detect possible intracellular staining, we performed antigen retrieval by boiling the sections with $10 \mathrm{mM}$ citric acid ( $\mathrm{pH}$ 6.0) for $10 \mathrm{~min}$ in a domestic microwave oven $(600 \mathrm{~W})$. Slides were allowed to cool down for $1 \mathrm{~h}$ and after a washing step, blocked, and incubated with primary antibodies at $4^{\circ} \mathrm{C}$ overnight (antibody dilutions: ABCG2: $1.25 \mu \mathrm{g} / \mathrm{ml}$; CD 10: 1:30 tissue culture supernatant; CK 19: $1 \mu \mathrm{g} / \mathrm{ml})$. Optimal antibody concentrations were determined by titrating serial antibody dilutions. The dilutions applied correspond to the minimum concentration revealing a fluorescence signal.

The following species-specific secondary antibodies were applied: Alexa Fluor ${ }^{\mathbb{R}} 488$ (green) and 568 (red) conjugated goat anti-mouse IgGs (Molecular Probes, Eugene, OR, USA) at recommended dilutions.

For double-staining experiments, TRITC-WGA was dissolved in PBS and applied at a final concentration of $20 \mu \mathrm{g} / \mathrm{ml}$ for $1 \mathrm{~h}$ at room temperature, after incubation of slides with the secondary antibody. Cell nuclei were stained with $0.5 \mu \mathrm{g} / \mathrm{ml}$ bisbenzimide/PBS (Hoechst 33342, Sigma). Slides were rinsed with distilled water, dried, and mounted with permanent Immuno-Fluore mounting medium (ICN Biomedicals, Inc., Aurora, OH, USA).

For different types of gallbladder samples, negative controls were obtained by omitting the first antibody or by replacing with irrelevant IgGs.

Experiments were also done on cryosections $(4 \mu \mathrm{m})$ from frozen specimens. After cutting with a microtome, sections were air dried for $1 \mathrm{~h}$ at room temperature. Sections were fixed for $7 \mathrm{~min}$ in acetone at room temperature. All subsequent immunofluorescence staining steps were done analogous to that described for paraffin-embedded tissue sections, but without the necessity of antigen retrieval.

Sections were viewed on an Axioplan 2 microscope (Carl Zeiss, Jena, Germany). Acquisition of images was performed using the Axiocam video camera and the Axiovision 3.1 software (Carl Zeiss Vision, Munich, Germany). In order to avoid background signals, the exposure times for the individual antibody staining were evaluated for a certain magnification and kept constant between the samples.

For evaluation of immunofluorescence staining, three different staining patterns were selected: (a) staining of the plasma membrane in $>70 \%$ of cells, (b) plasma membrane and cytoplasmic compartments in $>70 \%$ of cells, and (c) cytoplasmic staining (when membrane staining was absent in $>70 \%$ of cells).

\section{Statistical Evaluation}

Analysis of the subcellular ABCG2 staining pattern was performed using the $\chi^{2}$ test and the SPSS (SPSS, 
Inc. Chicago, IL, USA) package. A difference was considered statistically significant at $P<0.05$.

\section{Isolation of Gallbladder Epithelial Cells (GBEC) and Wortmannin Treatment}

For isolation of epithelial cells, normal gallbladder tissue was stored for a maximum of $2 \mathrm{~h}$ after the resection of the organ in Leibovitz L-15 cell culture medium supplemented with $10 \%$ fetal bovine serum (Invitrogen, Carlsbad, CA, USA) and 1\% penicillin/streptomycin (PAN Biotech, Aldenbach, Germany). Epithelial cells were isolated by digestion of gallbladder tissue with collagenase IV (690 U/ml; Sigma, St Louis, MO, USA) combined with hyaluronidase $(85 \mathrm{U} / \mathrm{ml}$; Sigma) in Leibovitz medium without serum for $12 \mathrm{~h}$ at $4^{\circ} \mathrm{C}$ and $1 \mathrm{~h}$ at $37^{\circ} \mathrm{C}$. Thereafter, cell aggregates were collected by centrifugation at $1500 \times g$ for $10 \mathrm{~min}$. Epithelial origin of cells was proven by immunofluorescence staining with the anti-CK19 antibody.

From a normal gallbladder, GBEC were freshly isolated and incubated at $37^{\circ} \mathrm{C}$ with 10 and $100 \mathrm{nM}$ of the PI3K inhibitor wortmannin for $1 \mathrm{~h}$, respectively. Control GBEC were incubated without wortmannin in the presence of the vehicle (DMSO). All incubations were performed in triplicate. Directly after the treatment of GBEC, cells were fixed in suspension by different methods (4\% paraformaldehyde for $30 \mathrm{~min}$; acetone for $7 \mathrm{~min}$, both at room temperature; methanol for $10 \mathrm{~min}$ and acetone for $1 \mathrm{~min}$ at $-20^{\circ} \mathrm{C}$ ), in order to exclude the possibility of artifact formation. Samples were permeabilized by $0.1 \%$ saponin prior to antibody application. Indirect immunofluorescence was performed as described above.

\section{Results}

\section{ABCG2 mRNA and Protein Expression in Human Gallbladder}

First, we investigated whether ABCG2 is present in human gallbladder. By application of ABCG2specific primers in RT-PCR experiments, significant expression of ABCG2 mRNA was found in samples from normal and cholelithiasis gallbladder as well as gallbladder adenocarcinoma. Studies were extended to extrahepatic bile ducts and cholangiocarcinoma (CCC), revealing also ABCG2 expression (Figure 1a).

Application of the monoclonal anti-ABCG2 antibody BXP-21 in Western blots clearly demonstrated that ABCG2 protein is present at considerable levels in normal, cholelithiasis, and malignant gallbladder as well as in extrahepatic bile ducts and CCC (Figure 1b). In all specimens, the amount of ABCG2 mRNA expression correlated well with the protein levels. PCR and Western blot analysis were done in whole tissue samples, but the high expression of CK19, a

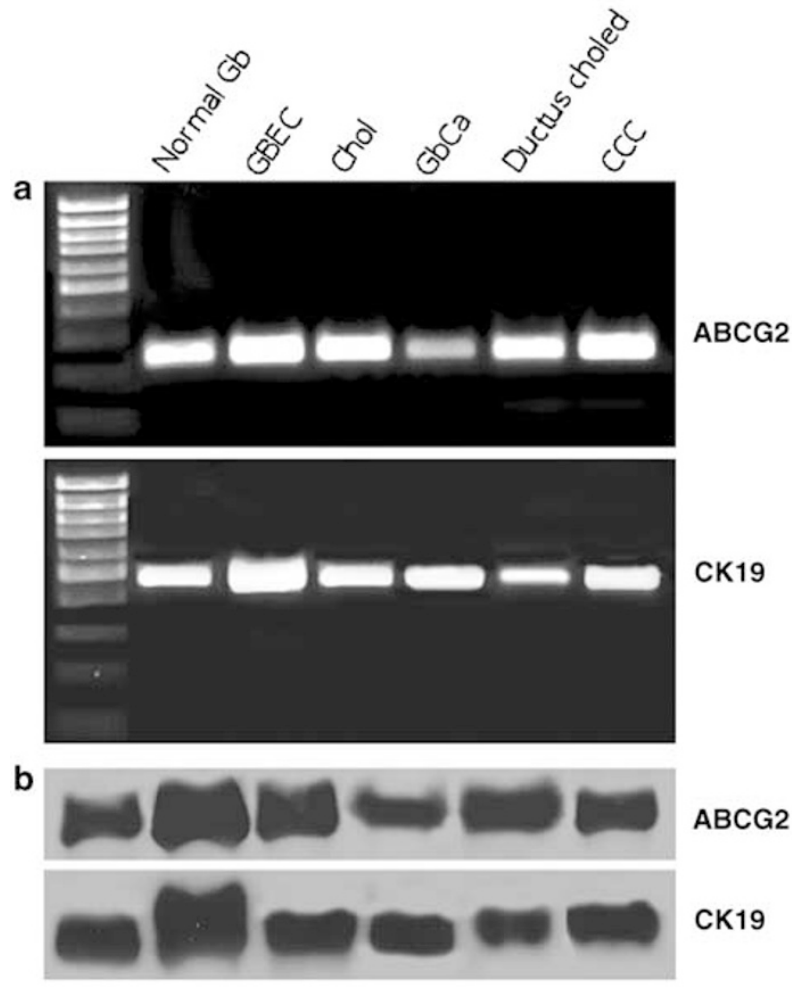

Figure 1 Analysis of ABCG2 and CK19 mRNA (a) and protein (b) expression in normal gallbladder (Gb), isolated gallbladder epithelial cells (GBEC), cholelithiasis (Chol), Gb adenocarcinoma (GbCa), extrahepatic bile duct (Ductus choled) and cholangiocarcinoma (CCC) specimens by RT-PCR and Western blotting. The amplicon size of ABCG2 was 231 and $433 \mathrm{bp}$ for cytokeratin (CK) 19 (a). A single immunoreactive band for ABCG2 was detected at approx. 70 and at $40 \mathrm{kDa}$ for CK 19 in all samples (b).

marker for the biliary epithelium, ${ }^{36}$ indicates that in the individual samples, a substantial part of RNA and protein used in the analysis is of epithelial origin. Therefore, it is likely that ABCG2 mRNA and protein are also derived from the biliary epithelium. This assumption was further confirmed by studies in freshly isolated gallbladder epithelial cells (GBEC) in which significant expression of ABCG2 mRNA and protein was found (Figure 1a and b).

\section{Immunolocalization of ABCG2 in Normal Human Gallbladder}

The subcellular distribution of ABCG2 was assessed by indirect immunofluorescence. Immunoreactivity of ABCG2 was seen at the luminal membrane of gallbladder epithelial cells (Figure 2), when the BXP-21 antibody was applied to either frozen (Figure 2a) or paraffin-embedded (Figure 2b) specimens derived from a normal gallbladder. Luminal staining is particularly obvious in the paraffin section in which the morphology is better conserved than in the frozen tissue section. No differences in the subcellular localization or in the degree of expression were observed after the formaldehyde- 

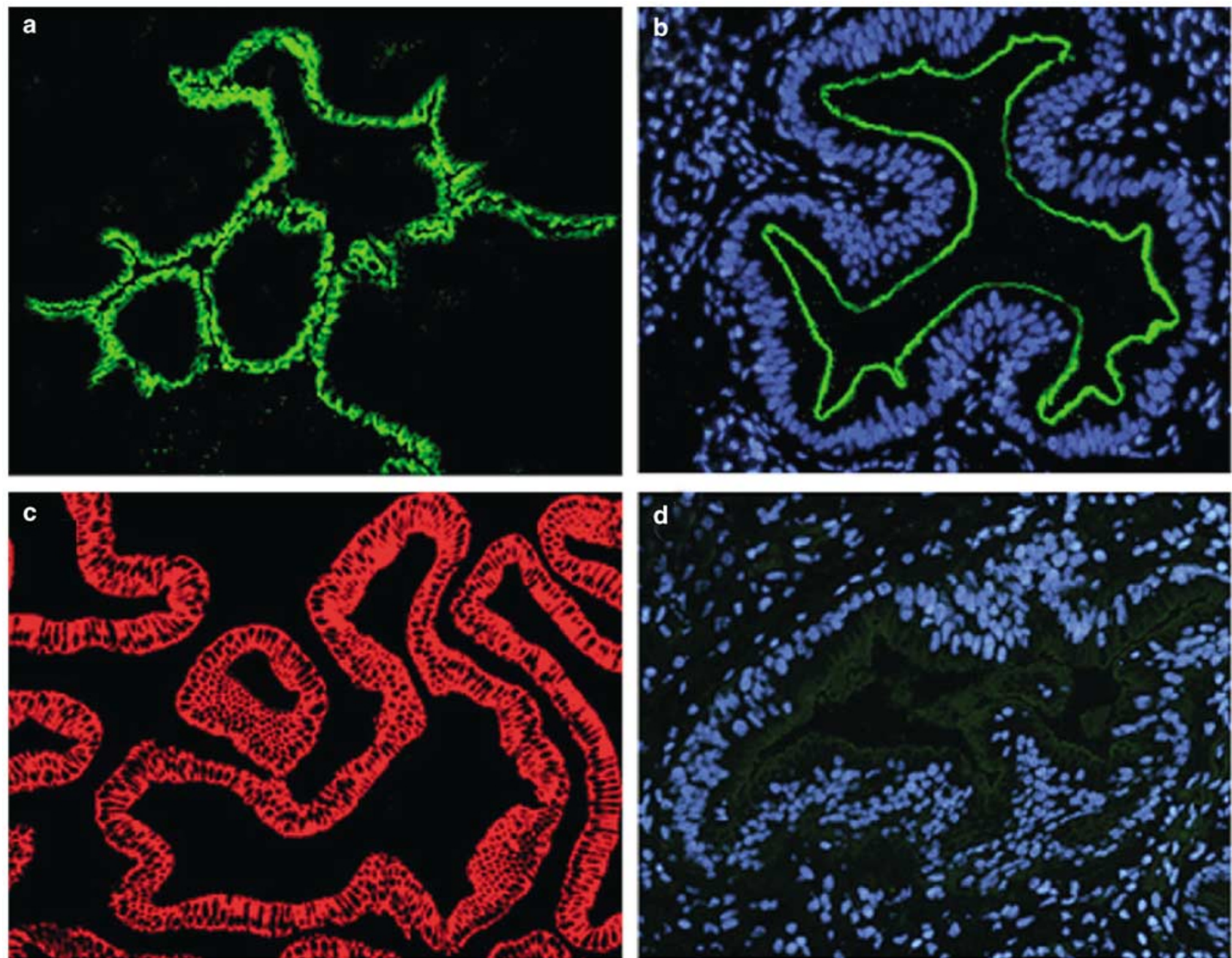

Figure 2 Subcellular localization of ABCG2 in gallbladder epithelium by indirect immunofluorescence. Green fluorescence indicates apical localized ABCG2 on a frozen (a) and a paraffin-embedded normal gallbladder section (b). Gallbladder epithelium is identified by red CK19 staining (c). The negative control (d) only displays blue fluorescence of cell nuclei. Original magnification: (a) $\times 100$, (b) $\times 200$, $(\mathbf{c}, \mathbf{d}) \times 125$.

fixation and antigen retrieval procedure, if samples were monitored with a given exposure time for a certain magnification. As demonstrated in Figure 2c, epithelial cells were identified by staining with the anti-CK19 antibody. In the negative controls, primary antibodies were replaced by irrelevant IgG. No ABCG2-derived staining was observed under these conditions. Only blue fluorescent nuclei stained with Hoechst dye are visible (Figure 2d).

\section{Subcellular Localization of ABCG2 in Diseased and Malignant Gallbladder}

Further indirect immunofluorescence experiments were performed to investigate the subcellular localization of ABCG2 in paraffin-embedded gallbladder sections from patients with cholelithiasis and adenocarcinomas of the gallbladder (Figures 3 and 4).
Using the monoclonal BXP-21 antibody, an Alexa 488-labeled secondary antibody, and the TRITClabeled lectin, wheat germ agglutinin (WGA), as an apical marker, ${ }^{37}$ we saw that in highly dedifferentiated tumor samples, the ABCG2 protein was located in the cytoplasm as well. Apical colocalization was monitored in cholelithiasis (Figure 3a) and gallbladder carcinoma specimens displaying high (Figure 3b) and poor (Figure 3c) differentiation grades. In the cholelithiasis and highly differentiated gallbladder carcinoma, the green ABCG2derived fluorescence is located at the apical membrane and overlaps with the red staining from the TRITC-labeled WGA resulting in a yellow to orange rim on the luminal side of epithelial cells (see last column, where epithelial cells can be recognized by the blue fluorescence of their nuclei).

In the poorly differentiated tumor, ABCG2 is additionally located in the cytoplasm of the majority of gallbladder epithelial cells. While its localization 

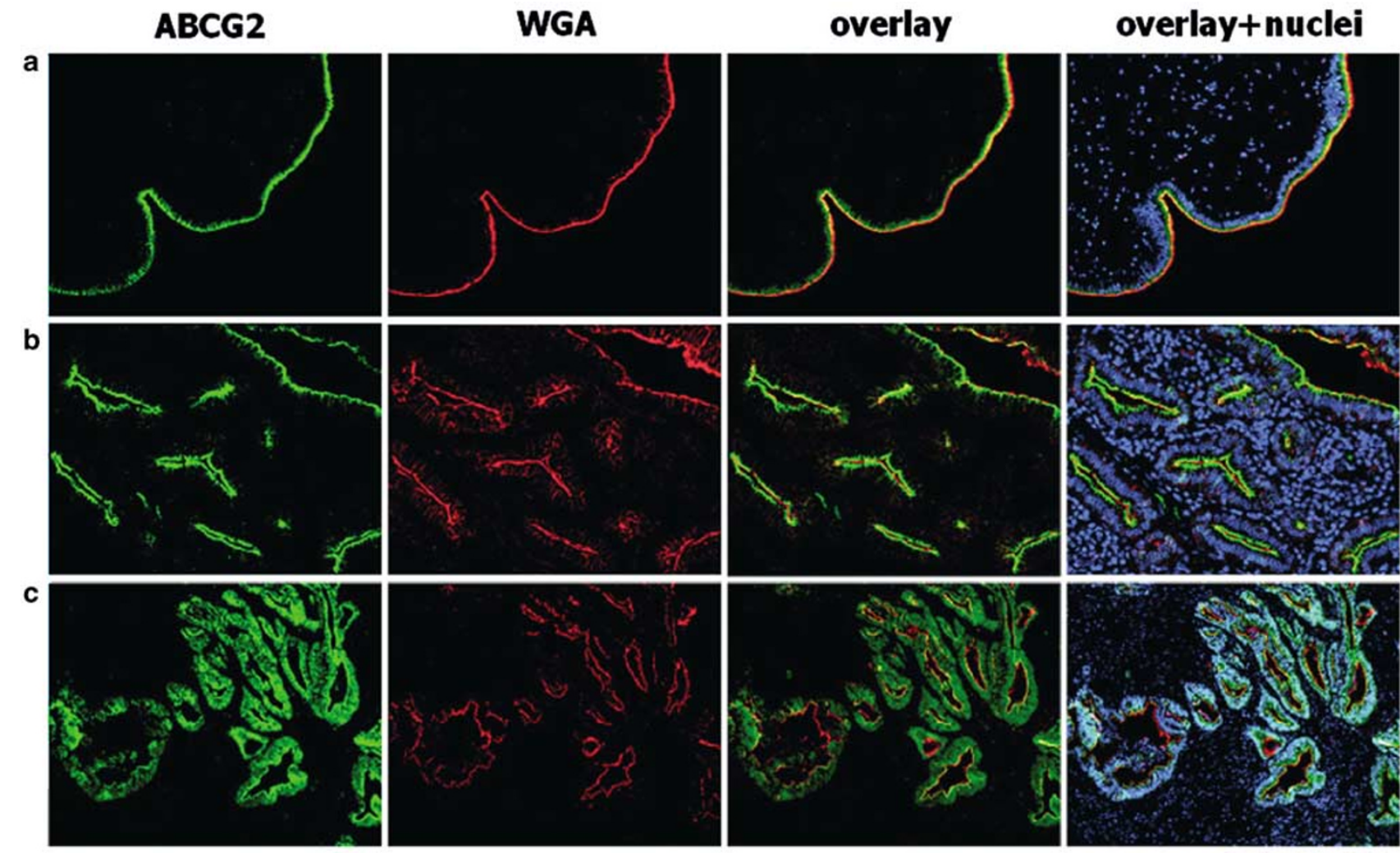

Figure 3 Apical colocalization of green ABCG2 immunofluorescence and red TRITC-labeled wheat germ agglutinin (WGA) on paraffin sections from human gallbladder specimens. Samples comprise cholelithiasis (a), a high- (b) and a low- (c) differentiated gallbladder carcinoma. Yellow staining indicates overlay of green ABCG2 and red TRITC-WGA fluorescence. Cell nuclei are stained blue with Hoechst 33342. Note that in the overlay picture of the low differentiated gallbladder carcinoma (c, overlay + nuclei), blue nuclei appear turquoise due to the green ABCG2 staining of the cell interior of epithelial cells. Original magnification: $(\mathbf{a}) \times 125$, (b) $\times 200$, (c) $\times 100$.

in the luminal membrane is again given by the orange to yellow color in the overlay picture, the green intracellular ABCG2 staining becomes prominent inside the epithelial cells. This is also indicated by the turquoise nuclei in the last column (last picture), where the blue-stained nuclei and the green ABCG2 fluorescence overlap.

In Figure 4, we show additional studies on the subcellular localization of ABCG2 in grade (G) 1, 2, and 3 tumors and a comparison with ABCG2 localization in cholelithiasis. Representative tumor samples with high expression of ABCG2 (green fluorescence) from each histological grade and a cholelithiasis sample are displayed in the left picture row. Again, in the cholelithiasis specimen, ABCG2 is found in the apical membrane. This was true for all cholelithiasis samples investigated $(n=10)$, and the ABCG2 staining pattern is not different from the pattern observed in the normal gallbladder (compare Figure 2).

Also, while ABCG2-derived fluorescence is found in the membrane in the G1 carcinoma specimen, localization in the intracellular space in addition to membrane staining is seen in dedifferentiated tumors of G2 and G3. Particularly, in the G3 tumor, membranous staining is hardly observable, while fluorescence in the intracellular space is prominent.
This raises the question of whether in dedifferentiated tumors, in addition to ABCG2, a marker enzyme of the apical gallbladder membrane, the neutral endopeptidase, ${ }^{7}$ might vanish from the membrane and be located in the intracellular space. As demonstrated in the right picture row of Figure 4, the red fluorescence signal remains membranous (apical membrane). However, in the grade 3 tumor, due to the distraction of the normal architecture, neutral endopeptidase staining could not be defined as luminal or apical.

\section{Gallbladder Carcinoma Patients}

In total, we investigated 41 gallbladder carcinoma patients with a median age of 69 years and with different tumor grades and stages. Clinical data are summarized in Table 1 and indicate the poor prognosis of this malignancy even after a complete resection of the tumor. This was also observed previously. ${ }^{2}$ In our study, longest median survival is 54.7 and 52 months and it is seen in patients with well-differentiated tumors (histological G1 and T1a and $1 \mathrm{~b}$ ). For patients with poorly differentiated (G3), advanced $(\mathrm{T} 3,4)$ tumors, the survival period is reduced to less than 10 months. To take these data 

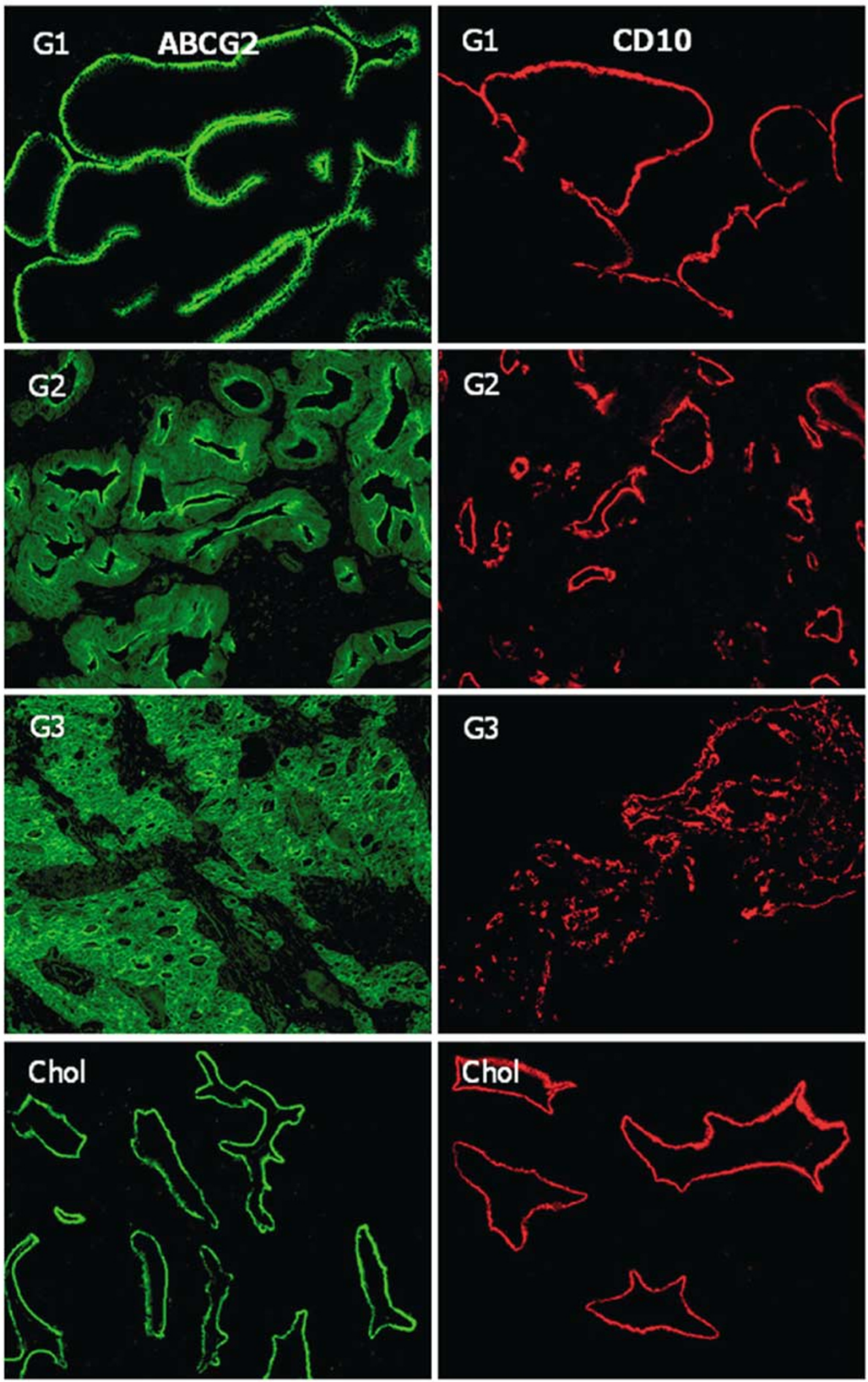

Figure 4 Immunolocalization of ABCG2 (left pictures, green fluorescence) and the apical marker neutral endopeptidase (CD10) (right pictures, red fluorescence) in representative specimens from patients with gallbladder adenocarcinoma grade 1,2, and 3 (G1-3) and a cholelithiasis patient (Chol). While in the grade 1 tumor and cholelithiasis specimens, ABCG2 staining is prominent at the luminal membrane, additional cytosolic staining occurs in dedifferentiated grade 2 and 3 samples. CD10 is membrane-localized independent on the degree of dedifferentiation. Original magnification: $(\mathrm{G} 1) \times 160$, (G2) $\times 125$, (G3) $\times 100$, (Chol, left picture) $\times 100$, (Chol, right picture) $\times 160$ 
Table 2 ABCG2 expression pattern and its clinicopathologic correlation in gallbladder carcinomas

\begin{tabular}{|c|c|c|c|c|}
\hline T classification & No. & Membrane & $\begin{array}{l}\text { Membrane } \\
\text { and } \\
\text { cytoplasm }\end{array}$ & Cytoplasm \\
\hline Grade 1 & 20 & 20 & 0 & 0 \\
\hline T1a & 6 & 6 & 0 & 0 \\
\hline $\mathrm{T} 1 \mathrm{~b}$ & 11 & 11 & 0 & 0 \\
\hline $\mathrm{T} 2$ & 2 & 2 & 0 & 0 \\
\hline T3 & 1 & 1 & 0 & 0 \\
\hline Grade 2 & 10 & 2 & 7 & 0 \\
\hline $\mathrm{T} 2$ & $8^{*}$ & 2 & 5 & 0 \\
\hline T3 & 1 & 0 & 1 & 0 \\
\hline $\mathrm{T} 4$ & 1 & 0 & 1 & 0 \\
\hline Grade 3 & 11 & 2 & 6 & 3 \\
\hline T1b & 1 & 1 & 0 & 0 \\
\hline $\mathrm{T} 2$ & 3 & 0 & 2 & 1 \\
\hline T3 & 3 & 0 & 1 & 2 \\
\hline $\mathrm{T} 4$ & 4 & 1 & 3 & 0 \\
\hline Total & 41 & 24 & 13 & 3 \\
\hline
\end{tabular}

Significance of subcellular localization in grade 1 vs grade 2 and 3 tumor samples: membrane localization $(P<0.01)$; localization in membrane and cytoplasm $(P<0.01)$; localization in cytoplasm $(P<0.01)$

${ }^{*}$ In one sample, ABCG2 could not be evaluated.

into consideration, we grouped the specimens according to the degree of differentiation (G1-G3) for the analysis of ABCG2 staining (Table 2).

In Table 2, the subcellular localization of ABCG2 in the carcinoma patients is summarized. Data are given with respect to grading and staging of tumors. In G1 tumors $(n=20)$, ABCG2 is located in the luminal membrane, and resembles the staining pattern observed in normal gallbladder and in cholelithiasis samples.

By contrast, in G2 tumors $(n=10)$, in seven samples, ABCG2 staining is still visible at the luminal membrane, but is also present in intracellular areas. In two samples, only membrane staining is observed and in one sample, ABCG2 is below the detection limit.

In highly dedifferentiated (G3) tumors $(n=11)$, the green fluorescence becomes intense within the cells, but is still additionally present on the membrane in six samples. In three specimens, ABCG2 is only still detectable in the cytoplasm. Remarkably, however, in two samples, it was still at the plasma membrane.

Subcellular localization of ABCG2 does not seem to relate to the tumor stage, as luminal staining was found in patients with well-differentiated (G1) tumors in both an early (T1) and advanced stage (T2-4). In patients with advanced tumors (T2-4), double localization (membrane and cytoplasmic compartments) is frequent, and this could be related to the fact that the majority of these tumors are also characterized by a poor histological grade (G2-3).

At a given time of exposure, the intensity of the green fluorescent signal indicating high or low ABCG2 expression levels varied within the indivi- dual gallbladder samples, but in the carcinoma samples, a definite trend regarding the tumor grade or stage could not be found. In contrast to normal and cholelithiasis samples, a majority of which displayed intense fluorescence, strong ABCG2 staining was only seen in 11 of 41 gallbladder carcinoma specimens.

\section{Cholangiocarcinoma}

To compare the ABCG2 staining pattern in gallbladder epithelium to that in other epithelia in the biliary tree, we monitored the subcellular localization of ABCG2 in proliferating bile ducts in cholangiocarcinoma specimens (CCC) (Figure 5). Proliferating bile ducts were identified by the expression of CK19 (Figure 5, right pictures). ${ }^{38}$ ABCG2 is present in the apical membrane of normal bile ductular cells (see insert), but is found in the cytosol in addition to the membrane in CCC cells of G2 and G3 tumors (Figure 5, left pictures).

\section{Translocation of ABCG2 in GBEC}

To investigate whether membrane localization of ABCG2 in gallbladder epithelium might be sensitive to the inhibition of phosphatidylinositol 3-kinase (PI3K), we isolated gallbladder epithelial cells and studied the influence of the PI3K inhibitor wortmannin on the translocation of ABCG2 by indirect immunofluorescence. Application of wortmannin in concentrations that do not inhibit kinases others than PI3K (10 and $100 \mathrm{nM}$ wortmannin for $1 \mathrm{~h}$ ) caused a pronounced ABCG2 staining in the intracellular compartment, visible as vesicular structures in the cytoplasm in these cells. Membrane staining for ABCG2, as clearly visible in the control GBEC, is no longer present. This indicates that membrane localization is sensitive to PI3K inhibition (Figure 6, green fluorescence). Again, CK19 served as an epithelial marker (Figure 6, red fluorescence).

\section{Discussion}

In the present study, the expression of the ABCG2 efflux pump was demonstrated in healthy and diseased gallbladder epithelium and gallbladder adenocarcinoma in 54 human samples. ABCG2 is located at the luminal plasma membrane of epithelial cells in normal, cholelithiasis, and well-differentiated carcinomas of the gallbladder, while in advanced gallbladder adenocarcinomas with poor histological grades, both, membrane and cytoplasmic ABCG2 immunoreactivity was observed in the majority of specimens. Additional studies in isolated gallbladder epithelial cells indicated that membrane insertion of the transporter is sensitive to PI3K inhibition. 

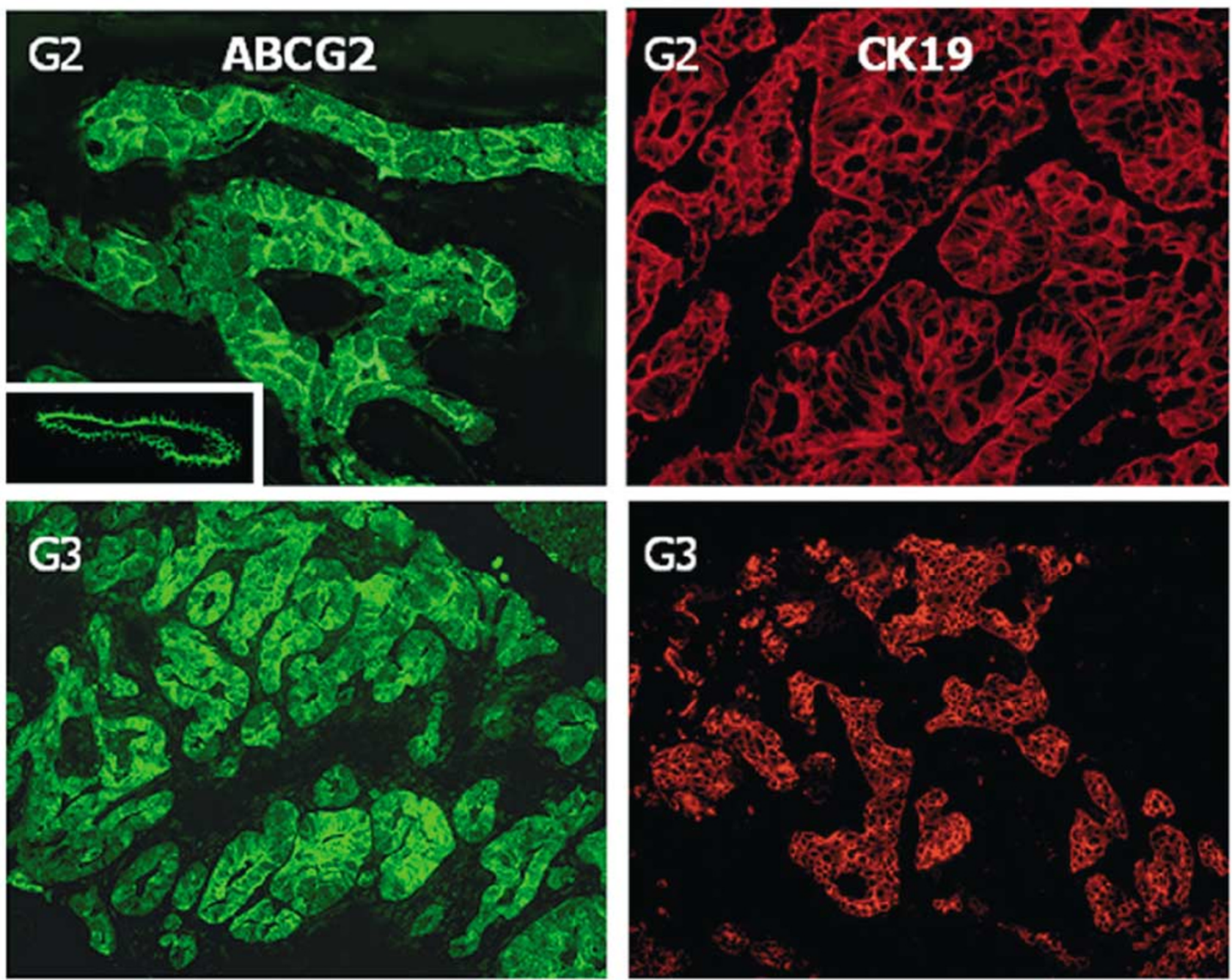

Figure 5 Immunofluorescence staining of ABCG2 and cytokeratin (CK) 19 in cholangiocarcinoma (CCC) grade 2 (G2) and grade 3 (G3). Green fluorescence indicates membranous and additional cytoplasmic ABCG2 staining in the advanced grade 2 and 3 tumors. Luminal localization of ABCG2 in a normal human liver bile duct is displayed in the insert. Identification of proliferating bile ductular cells was performed by red CK19 staining (right pictures). Original magnification: (G2) × 250, (insert) $\times 250$, (G3, left picture) $\times 125$, (G3, right picture) $\times 100$.

The pronounced expression of ABCG2 in the luminal membrane of gallbladder epithelial cells is evidence of the important role of this efflux pump. It is likely that it mediates protection from xenotoxins, drugs, porphyrins, as well as from degradation products from various endogenous and exogenous sources present in high concentrations in bile. It also points to the importance of the biliary tract epithelium, where specialized mechanisms are active in the modification of primary hepatic bile. ${ }^{39}$

In gallbladder as well as in other parts of the biliary tract, not only ABC-transporters such as ABCG2 form an efficient barrier, but also phase I and II drug-metabolizing enzymes, which are heterogenously distributed in the biliary tract epithelium, cooperate to support the excretion of various xenotoxins by the formation of more water-soluble and less active compounds. ${ }^{40}$ However, it should also be kept in mind that biotransformation enzymes can create potentially harmful metabolites and carcinogens. In this case, ATP-dependent efflux pumps are urgently required to prevent further injuries in the biliary tract.

ABCG2 could also be involved in the transport of sterols. The transporter might assist ABCG5/ABCG8 to prevent excessive cholesterol absorption into the gallbladder epithelium, particularly under conditions, where cholesterol concentrations in gallbladder are rising, for example, in the interdigestive phase. ${ }^{24}$ As neither a difference in the expression nor in the cellular localization of ABCG2 in normal, cholelithiasis, and well-differentiated adenocarcinomas was observed in our study, ABCG2 is unlikely to participate in the pathogenesis of cholelithiasis and gallbladder carcinoma with respect to common risk factors. ${ }^{1,41}$

An important finding in our study was that in advanced gallbladder carcinoma tissue, and also in 


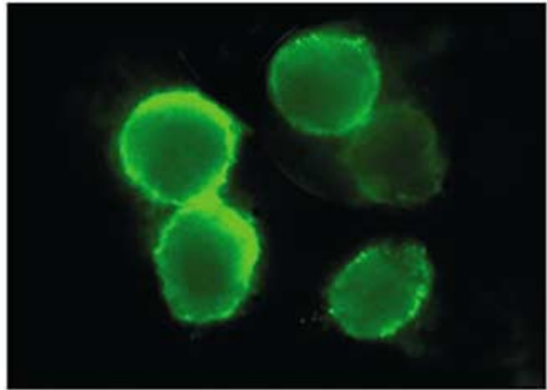

No treatment ABCG2

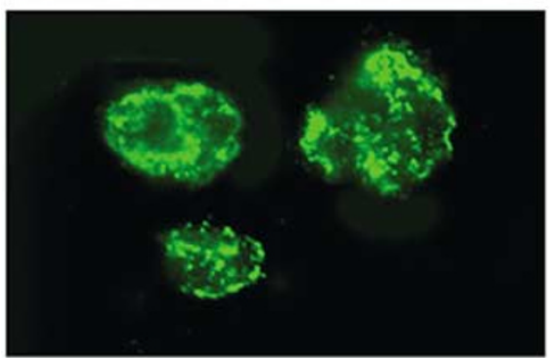

Wortmannin ABCG2

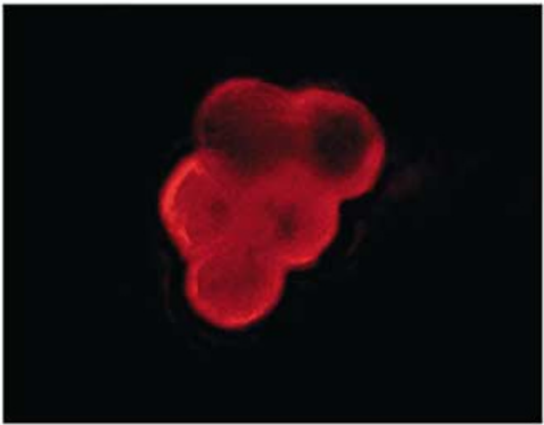

Epithelial marker CK19

Figure 6 ABCG2 translocation in isolated gallbladder epithelia cells (GBEC) by wortmannin. GBEC were isolated from normal gallbladder and cultured in the absence or presence of $100 \mathrm{nM}$ wortmannin for $1 \mathrm{~h}$. ABCG2 was detected by the BXP-21 antibody either on the membrane in untreated cells or in intracellular vesicles in treated cells. Expression of the epithelial marker CK19 proved the epithelial origin of isolated GBEC. Original magnification: $\times 800$.

poorly differentiated CCC, ABCG2 is located in the intracellular compartment in addition to the membrane. The decline of membrane localized ABCG2 in these poorly differentiated carcinomas might suggest that ABCG2 is no longer working as a plasma membrane efflux pump in these tumors. This assumption is supported by data of a recent study in mice primary bronchial smooth muscle cells, where ABCG2 located in the cytoplasm does not efflux Hoechst dye 33342, a well-known ABCG2 substrate. ${ }^{42}$ Also, impaired membrane localization caused by single-nucleotide polymorphisms results in a decline of ABCG2 ATPase activity. ${ }^{43}$ Whether this applies to ABCG2 function in the in vivo situation must be established.

In any case, adenocarcinomas of the gallbladder and other tumors of the biliary tract are characterized by a high degree of intrinsic chemoresistance against various chemotherapy regimens. ${ }^{44}$ So far, significant responses to the chemotherapy of gallbladder carcinoma were only reported from a combined therapy with two non-ABCG2 substrates, gemcitabine and cisplatin. ${ }^{2}$

All gallbladder carcinomas tested in this study were derived from patients without prior exposure to chemotherapy. Therefore, intracellular ABCG2 staining is not a result of the medication. As the localization of the neutral endopeptidase is not affected by dedifferentiation, ABCG2 translocalization may be caused by a selective cellular signaling pathway. In polarized cells, for example, hepatocytes, newly synthesized ABC-transporters of the apical membrane are either delivered directly from the Golgi to the apical pole or inserted into the basolateral membrane before being redistributed to the apical side by transcytosis. ${ }^{45}$ PI3K has been implicated in the regulation of the insertion of ATPdependent transport proteins into the canalicular membrane of hepatocytes. This process is sensitive to the inhibition of the kinase by wortmannin. ${ }^{34}$ Analogous to its effect on transporter insertion in hepatocytes, in biliary epithelial cells, PI3K signaling regulates membrane transport processes (ATPand chloride efflux) as well. ${ }^{46}$ Additionally, in hematopoetic stem cell side populations in mice, PI3K-Akt-mediated regulation of ABCG2 expression might be a mechanism by which stem cells are protected from naturally occurring genotoxic compounds. ${ }^{33}$

We demonstrate by indirect immunofluorescence in isolated GBEC that the application of wortmannin results in ABCG2 translocation into intracellular vesicles, indicating that PI3K signaling is also important for ABCG2 membrane insertion in gallbladder epithelium. As PI3K controls the reorganization of the actin cytoskeleton during the course of malignant transformation processes, membrane loss of ABCG2 might coincide with a loss of interaction between ABCG2 and ezrin-radixin-moesin (ERM) protein complexes and PDZ-domains at the membrane-cytoskeleton interface. An analogous mechanism was demonstrated for the ABC-transporter mrp2 in the hepatocyte canalicular membrane. ${ }^{47}$ While the ERM protein radixin is present in hepatocytes, another ERM-protein, ezrin, is highly expressed in bile ductular cells. ${ }^{48}$

In summary, the significant expression of ABCG2 in gallbladder epithelial cells suggests a protective role for ABCG2 in this organ. Disturbed PI3K signaling during tumor progression might explain the altered subcellular localization of ABCG2 in samples from patients with poorly differentiated gallbladder carcinomas.

\section{Acknowledgements}

This work was supported by the Jubilaeumsfonds of the Austrian National Bank P8249 (OP) and P9894 (JW). AS received a fellowship from the Austrian Academy of Sciences (DOC Program). Color prints were supported by DAKO, Vienna, Austria. 


\section{References}

1 Beckingham IJ. ABC of diseases of liver, pancreas, and biliary system. Gallstone disease. BMJ 2001;322:91-94.

2 Misra S, Chaturvedi A, Misra NC, et al. Carcinoma of the gallbladder. Lancet Oncol 2003;4:167-176.

3 Bodo A, Bakos E, Szeri F, et al. The role of multidrug transporters in drug availability, metabolism and toxicity. Toxicol Lett 2003;140-141:133-143.

4 Elferink RO, Groen AK. Genetic defects in hepatobiliary transport. Biochim Biophys Acta 2002;1586: 129-145.

5 Faber KN, Muller M, Jansen PL. Drug transport proteins in the liver. Adv Drug Deliv Rev 2003; 55:107-124.

6 Alpini G, McGill JM, Larusso NF. The pathobiology of biliary epithelia. Hepatology 2002;35:1256-1268.

7 Scoazec JY, Bringuier AF, Medina JF, et al. The plasma membrane polarity of human biliary epithelial cells: in situ immunohistochemical analysis and functional implications. J Hepatol 1997;26:543-553.

8 Rost D, König J, Weiss G, et al. Expression and localization of the multidrug resistance proteins MRP2 and MRP3 in human gallbladder epithelia. Gastroenterology 2001;121:1203-1208.

9 Gigliozzi A, Fraioli F, Sundaram P, et al. Molecular identification and functional characterization of Mdr1a in rat cholangiocytes. Gastroenterology 2000;119:1113-1122.

10 Doyle LA, Yang W, Abruzzo LV, et al. A multidrug resistance transporter from human MCF-7 breast cancer cells. Proc Natl Acad Sci USA 1998;95: 15665-15670.

11 Maliepaard M, Scheffer GL, Faneyte IF, et al. Subcellular localization and distribution of the breast cancer resistance protein transporter in normal human tissues. Cancer Res 2001;61:3458-3464.

12 Shimano K, Satake M, Okaya A, et al. Hepatic oval cells have the side population phenotype defined by expression of ATP-binding cassette transporter ABCG2/BCRP1. Am J Pathol 2003;163:3-9.

13 Krishnamurthy P, Ross DD, Nakanishi T, et al. The stem cell marker Bcrp/ABCG2 enhances hypoxic cell survival through interactions with heme. J Biol Chem Mar 2004;24, [Epub ahead of print].

14 Litman T, Druley TE, Stein WD, et al. From MDR to MXR: new understanding of multidrug resistance systems, their properties and clinical significance. Cell Mol Life Sci 2001;58:931-959.

15 Allen JD, Schinkel AH. Multidrug resistance and pharmacological protection mediated by the breast cancer resistance protein (BCRP/ABCG2). Mol Cancer Ther 2002;1:427-434.

16 Dietrich CG, Geier A, Oude Elferink RP. ABC of oral bioavailability: transporters as gatekeepers in the gut. Gut 2003;52:1788-1795.

17 Imai Y, Asada S, Tsukahara S, et al. Breast cancer resistance protein exports sulfated estrogens but not free estrogens. Mol Pharmacol 2003;64:610-618.

18 Ee PL, Kamalakaran S, Tonetti D, et al. Identification of a novel estrogen response element in the breast cancer resistance protein (ABCG2) gene. Cancer Res 2004;64:1247-1251.

19 Jonker JW, Buitelaar M, Wagenaar E, et al. The breast cancer resistance protein protects against a major chlorophyll-derived dietary phototoxin and protoporphyria. Proc Natl Acad Sci USA 2002;99:15649-15654.
20 Robey RW, Steadman K, Polgar O, et al. Pheophorbide a is a specific probe for ABCG2 function and inhibition. Cancer Res 2004;64:1242-1246.

21 Herwaarden AE, Jonker JW, Wagenaar E, et al. The breast cancer resistance protein (Bcrp1/Abcg2) restricts exposure to the dietary carcinogen 2-amino-1methyl-6-phenylimidazo[4,5-b]pyridine. Cancer Res 2003;63:6447-6452.

22 Serrano MA, Bayon JE, Pascolo L, et al. Evidence for carrier-mediated transport of unconjugated bilirubin across plasma membrane vesicles from human placental trophoblast. Placenta 2002;23:527-535.

23 Janvilisri T, Venter H, Shahi S, et al. Sterol transport by the human breast cancer resistance protein (ABCG2) expressed in lactococcus lactis. J Biol Chem 2003;278:20645-20651.

24 Tauscher A, Kuver R. ABCG5 and ABCG8 are expressed in gallbladder epithelial cells. Biochem Biophys Res Commun 2003;307:1021-1028.

25 Woehlecke $\mathrm{H}$, Pohl A, Alder-Baerens $\mathrm{N}$, et al. Enhanced exposure of phosphatidylserine in human gastric carcinoma cells overexpressing the half-size ABC transporter BCRP (ABCG2). Biochem J 2003;376: 489-495.

26 Rocchi E, Khodjakov A, Volk EL, et al. The product of the ABC half-transporter gene ABCG2 (BCRP/MXR/ $\mathrm{ABCP})$ is expressed in the plasma membrane. Biochem Biophys Res Commun 2000;271:42-46.

27 Doyle LA, Ross DD. Multidrug resistance mediated by the breast cancer resistance protein BCRP (ABCG2). Oncogene 2003;22:7340-7358.

28 Diestra JE, Scheffer GL, Catala I, et al. Frequent expression of the multi-drug resistance-associated protein BCRP/MXR/ABCP/ABCG2 in human tumours detected by the BXP-21 monoclonal antibody in paraffin-embedded material. J Pathol 2002;198: 213-219.

29 Scheffer GL, Maliepaard M, Pijnenborg AC, et al. Breast cancer resistance protein is localized at the plasma membrane in mitoxantrone- and topotecanresistant cell lines. Cancer Res 2000;60:2589-2593.

30 Diestra JE, Condom E, Del Muro XG, et al. Expression of multidrug resistance proteins P-glycoprotein, multidrug resistance protein 1 , breast cancer resistance protein and lung resistance related protein in locally advanced bladder cancer treated with neoadjuvant chemotherapy: biological and clinical implications. J Urol 2003;170:1383-1387.

31 Kawabata S, Oka M, Soda H, et al. Expression and functional analyses of breast cancer resistance protein in lung cancer. Clin Cancer Res 2003;9: 3052-3057.

32 Faneyte IF, Kristel PM, Maliepaard M, et al. Expression of the breast cancer resistance protein in breast cancer. Clin Cancer Res 2002;8:1068-1074.

33 Mogi M, Yang J, Lambert JF, et al. Akt signaling regulates side population cell phenotype via Bcrp1 translocation. J Biol Chem 2003;278:39068-39075.

34 Misra S, Ujhazy P, Varticovski L, et al. Phosphoinositide 3-kinase lipid products regulate ATP-dependent transport by sister of P-glycoprotein and multidrug resistance associated protein 2 in bile canalicular membrane vesicles. Proc Natl Acad Sci USA 1999;96:5814-5819.

35 Wittekind Ch, Meyer HJ, Bootz F. TNM-Klassifikation maligner Tumoren, 6th edn. Berlin - Heidelberg - New York: Springer Verlag, 2003. 
36 Lee MJ, Lee HS, Kim WH, et al. Expression of mucins and cytokeratins in primary carcinomas of the digestive system. Mod Pathol 2003;16:403-410.

37 Torres RJ, Altenberg GA, Copello JA, et al. Preservation of structural and functional polarity in isolated epithelial cells. Am J Physiol 1996;270:C1864-C1874.

38 Shimonishi T, Miyazaki K, Nakanuma Y. Cytokeratin profile relates to histological subtypes and intrahepatic location of intrahepatic cholangiocarcinoma and primary sites of metastatic adenocarcinoma of liver. Histopathology 2000;37:55-63.

39 Igimi H, Yamamoto F, Lee SP. Gallbladder mucosal function: studies in absorption and secretion in humans and in dog gallbladder epithelium. Am J Physiol 1992;263:G69-G74.

40 Lakehal F, Wendum D, Barbu V, et al. Phase I and phase II drug-metabolizing enzymes are expressed and heterogeneously distributed in the biliary epithelium. Hepatology 1999;30:1498-1506.

41 Dowling RH. Review: pathogenesis of gallstones. Aliment Pharmacol Ther 2000;14(Suppl 2):39-47.

42 Summer R, Kotton DN, Sun X, et al. Side population cells and Bcrp1 expression in lung. Am J Physiol Lung Cell Mol Physiol 2003;285:L97-L104.
43 Mizuarai S, Aozasa N, Kotani H. Single nucleotide polymorphisms result in impaired membrane localization and reduced ATPase activity in multidrug transporter ABCG2. Int J Cancer 2004;109: 238-246.

44 Donohue JH. Present status of the diagnosis and treatment of gallbladder carcinoma. J Hepatobiliary Pancreat Surg 2001;8:530-534.

45 Kipp H, Pichetshote N, Arias IM. Transporters on demand: intrahepatic pools of canalicular ATP binding cassette transporters in rat liver. J Biol Chem 2001;276:7218-7224.

46 Feranchak AP, Roman RM, Doctor RB, et al. The lipid products of phosphoinositide 3-kinase contribute to regulation of cholangiocyte ATP and chloride transport. J Biol Chem 1999;274:30979-30986.

47 Kikuchi S, Hata M, Fukumoto K, et al. Radixin deficiency causes conjugated hyperbilirubinemia with loss of Mrp2 from bile canalicular membranes. Nat Genet 2003;31:320-325.

48 Fouassier L, Duan CY, Feranchak AP, et al. Ezrinradixin-moesin-binding phosphoprotein 50 is expressed at the apical membrane of rat liver epithelia. Hepatology 2001;33:166-176. 